\title{
Plant functional traits reveal the relative contribution of habitat and food preferences to the diet of grasshoppers
}

\author{
Sébastien Ibanez - Olivier Manneville $\cdot$ Christian Miquel $\cdot$ Pierre Taberlet $\cdot$ \\ Alice Valentini $\cdot$ Serge Aubert $\cdot$ Eric Coissac $\cdot$ Marie-Pascale Colace $\cdot$ \\ Quentin Duparc · Sandra Lavorel · Marco Moretti
}

Received: 13 August 2012 / Accepted: 9 July 2013 / Published online: 6 October 2013

(C) Springer-Verlag Berlin Heidelberg 2013

\begin{abstract}
Food preferences and food availability are two major determinants of the diet of generalist herbivores and of their spatial distribution. How do these factors interact and eventually lead to diet differentiation in co-occurring herbivores? We quantified the diet of four grasshopper species co-occurring in subalpine grasslands using DNA barcoding of the plants contained in the faeces of individuals sampled in the field. The food preferences of each grasshopper species were assessed by a choice (cafeteria) experiment from among 24 plant species common in five grassland plots, in which the four grasshoppers were collected, while the habitat was described by the relative abundance of plant species in the grassland plots. Plant species were
\end{abstract}

Communicated by Roland Brandl.

Electronic supplementary material The online version of this article (doi:10.1007/s00442-013-2738-0) contains supplementary material, which is available to authorized users.

S. Ibanez $(\bowtie) \cdot$ M. Moretti

Community Ecology Research Unit, Swiss Federal Research

Institute WSL, Via Belsoggiorno 22, 6500 Bellinzona,

Switzerland

e-mail: sebastien.ibanez@univ-savoie.fr

Present Address:

S. Ibanez

Laboratoire d'Écologie Alpine, UMR CNRS 5553, Université de

Savoie, 73376 Le Bourget-du-lac, France

O. Manneville $\cdot$ S. Aubert $\cdot$ M.-P. Colace $\cdot$ Q. Duparc Station Alpine Joseph Fourier, UMS CNRS 3370, Université Joseph Fourier, Grenoble, France

C. Miquel · P. Taberlet · A. Valentini - S. Aubert - E. Coissac · M.-P. Colace · Q. Duparc · S. Lavorel

Laboratoire d'Ecologie Alpine, UMR CNRS 5553, Université

Joseph Fourier, Grenoble, France characterised by their leaf economics spectrum (LES), quantifying their nutrient vs. structural tissue content. The grasshoppers' diet, described by the mean LES of the plants eaten, could be explained by their plant preferences but not by the available plants in their habitat. The diet differed significantly across four grasshopper species pairs out of six, which validates food preferences assessed in standardised conditions as indicators for diet partitioning in nature. In contrast, variation of the functional diversity (FD) for LES in the diet was mostly correlated to the FD of the available plants in the habitat, suggesting that diet mixing depends on the environment and is not an intrinsic property of the grasshopper species. This study sheds light on the mechanisms determining the feeding niche of herbivores, showing that food preferences influence niche position whereas habitat diversity affects niche breadth.

Keywords DNA barcoding - Cafeteria experiment . Niche partitioning $\cdot$ Herbivory $\cdot$ Interaction networks

\section{Introduction}

Herbivores play a major role in ecosystem structure and function, but the mechanisms leading to the composition of their diet are not yet fully understood. The diet of specialist herbivores is generally well explained by specific behavioural, morphological and biochemical adaptations between the herbivore species and its host plant (e.g. Rowell-Rahier 1984). In contrast, generalists rarely show specific adaptations to particular plant species and are able to consume dozens of plant species from numerous families (Joern 1979; Franzke et al. 2010). However, they do not consume all the plant species available in their habitat. Their diet is diversified but still somehow selective. What 
are the mechanisms that explain the diet, thus the plant species' selection of generalist herbivores?

Generalist grazers have well-defined nutritional requirements in terms of carbohydrates, protein and water intake (Simpson et al. 2004; Behmer 2009), and have developed cognitive abilities that allow them to taste and learn to accurately choose their food (Bernays and Bright 2005). In the case of generalisation at the individual level, individuals can even mix different diets to achieve optimal quality and dilute toxins (Bernays et al. 1994; Unsicker et al. 2008). As a consequence, the choice of suitable food generally leads to a higher fitness (Simpson et al. 2004; Unsicker et al. 2008). When confronted with natural plant material, generalist grazers show marked preferences for certain plant species (Bernays and Chapman 1994; Pérez-Harguindeguy et al. 2003). Furthermore, generalist grazers need a balanced diet rather than a few particular plant species (Behmer and Joern 2008; Franzke et al. 2010), and more specifically a diverse set of plants that belong to different functional groups rather than just a large number of different plant species that belong to the same functional groups (Specht et al. 2008). Therefore, a functional approach is better suited than a taxonomical approach to describe the diet of herbivores, because it provides quantitative information on those plant functional characteristics that influence diet selection. Moreover, a functional approach using field data can build a bridge between experimental studies dealing with artificial food with contrasting nutritional properties that reflect plant traits, and field studies basically dealing with taxonomical data. Studies at plant species level have shown that leaf nitrogen content (LNC), leaf water content (the inverse of leaf dry matter content; LDMC) and specific leaf area (SLA) were associated with increased levels of herbivory by native invertebrates in central Argentina (Pérez-Harguindeguy et al. 2003), and that the palatability of plants to the house cricket Acheta domesticus increased with LNC and SLA, and decreased with LDMC, leaf carbon content (LCC) and C/N ratio (Schädler et al. 2003). However, as far as we know, such effects of plant traits on herbivore diets have not been tested at the community level.

Given this fundamental knowledge, we expect the diet of herbivores in plant communities under natural conditions to have biochemical properties similar to those of the food preferences of herbivores. For example, Pérez-Harguindeguy et al. (2003) found that herbivory in the field was correlated with the feeding preferences of snails and grasshoppers observed in a cafeteria experiment, although this study did not distinguish the herbivore species responsible for the observed consumption. In the context of a community of herbivores, we predict that species with contrasting nutritional requirements (Behmer and Joern 2008) should prefer different plant traits and hence have differentiated diets, possibly leading to niche partitioning (Sword et al. 2005) (prediction no. 1). At the same time, since the amount of a plant species eaten by herbivores generally correlates to its abundance in nature (Bernays and Chapman 1970; Ogden 1976; Speiser and Rowell-Rahier 1991; Singer and Stireman 2001) and as the functional traits of the plants available in the habitat may not necessarily match the functional traits of herbivores' preferred plant species, we expect that habitat and preferences may not necessarily have consistent effects on the functional make up of the diet of the herbivores (prediction no. 2).

To test these predictions, we studied a community of generalist grazing Gomphocerinae grasshoppers in subalpine grassland communities in the Central French Alps and assessed the interplay between their food preferences, habitat and diet (Fig. 1). We determined the diet of four Gomphocerinae grasshopper species in five contrasting plant communities by analysing DNA remains found in the faeces (Valentini et al. 2009), while their food preferences were established in standardised cafeteria experiments, where insects could choose leaves independently of plant architecture (Pérez-Harguindeguy et al. 2003). Finally the grasshoppers' habitat was characterised by the abundances of available plant species in each community where the insects were sampled. Gomphocerinae grasshoppers eat plants that belong to as many as ten to 20 families, mostly Poaceae, a few Fabaceae and some other dicotyledonous forbs (Joern 1979; Franzke et al. 2010). Gomphocerinae grasshoppers are therefore a potentially heterogeneous group with respect to diet. We aim to test whether differences in the diet of the four species can be explained by their preferences based on specific plant traits and/or by the traits of the available plants in their habitat.

\section{Materials and methods}

\section{General framework}

We characterised the diet of the four grasshopper species by the functional spectrum of ingested plants. This spectrum was quantified for each of the traits considered as relevant to the diet using the mean trait value across consumed species weighted by their relative abundance in the faeces (community weighed mean; CWM) (Garnier et al. 2004; Lavorel et al. 2008) and the variation of trait values (functional diversity; FD) (Laliberté and Legendre 2010). The diet CWM reflects the nutritional mixture that is ultimately digested by the herbivore and can be understood as the mean niche position, while the diet FD reflects the diet mixing process and can be understood as the niche breadth. In the same way, we characterised the food preferences by the functional spectrum of the preferred plant species, and the habitat by the functional spectrum of the available 


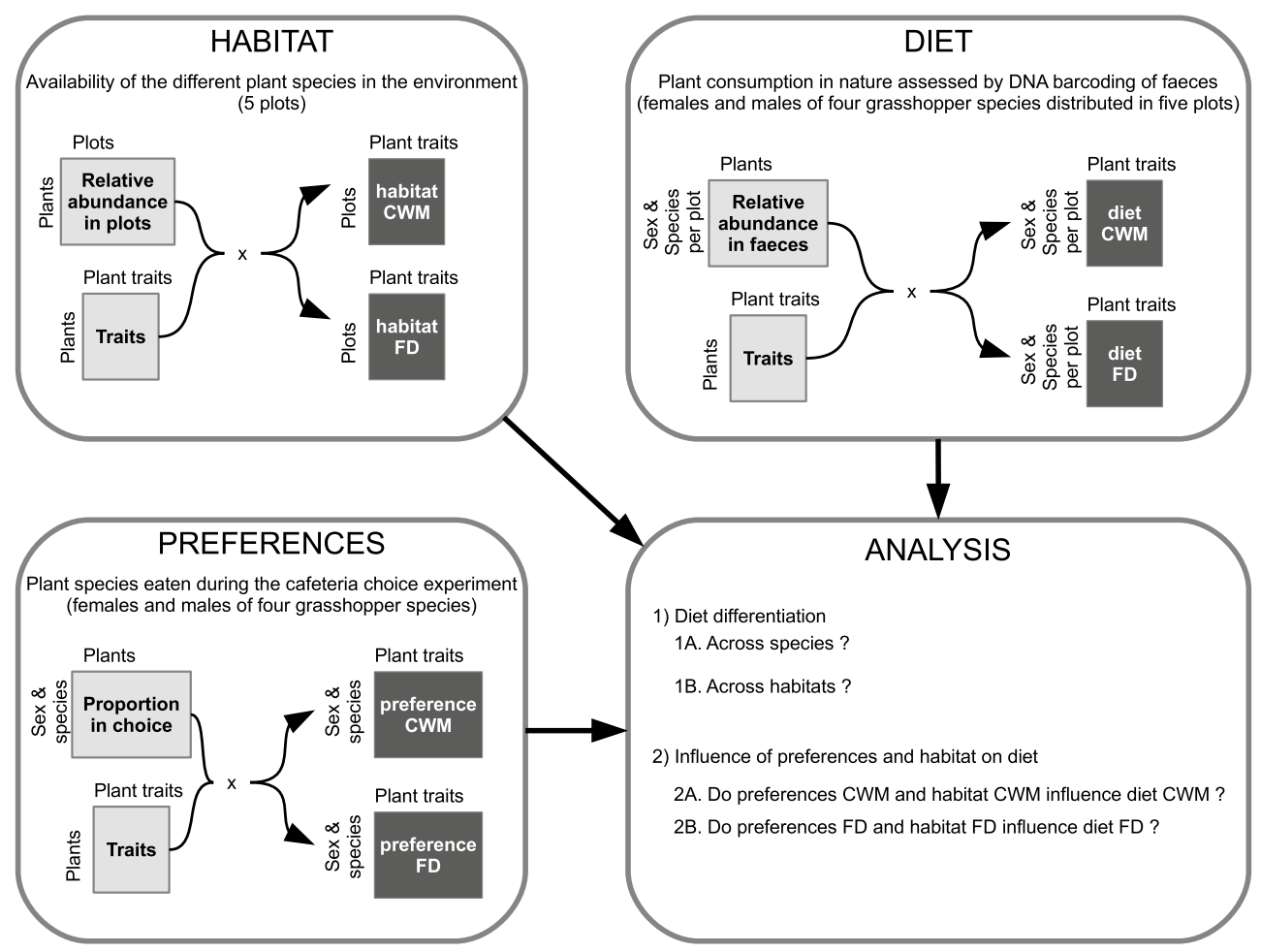

Fig. 1 Conceptual framework of the study, terms used and methods overview. The community weighted mean $(C W M)$ was calculated following: $\mathrm{CWM}=\sum_{i=1}^{n} p_{i} \times$ trait $_{i}$, where $n$ is the total number of plant species in the habitat (respectively the preferences, respectively the diet), $p_{i}$ the relative abundance of species $i$ in the habitat (respectively the preferences, respectively the diet) and trait ${ }_{i}$, the trait value of species $i$. The functional diversity $(F D)$ was calculated as the mean distance of the species' traits to the community weighted mean
(CWM). CWM measures the niche position while FD measures niche breadth. The habitat is characterised by the CWM and FD of the traits of the plant species present in the five plots studied. Preferences are characterised by the CWM and FD of the traits of the plants eaten in the cafeteria experiment by females and males of four grasshopper species $(2 \times 4=8$ rows). The diet is characterised by the CWM and FD of the traits of the plants found in the faeces of females and males of four grasshopper species, in each of the five plots plants in the field. Both were quantified using CWM and FD for each of the relevant traits using the relative amount of plants eaten in the cafeteria experiment and that found in the faeces, and plant species relative abundances in the plots, respectively. The diet and preferences of males and females were treated separately because reproducing females do not usually have the same nutritional requirements as males (Asshoff and Hattenschwiler 2005). We focused on six traits describing the quality and quantity of plant material: SLA, LDMC, LNC, LCC, leaf phosphorus content (LPC) and vegetative plant height (VPH). The leaf economics spectrum (LES) (Wright et al. 2004), defined by the first axis of a principal component analysis (PCA) of SLA, LDMC and LNC, reflects the functional trade-off between fibre and nutrients in leaf tissue and thus nutritional value (Pontes et al. 2007), and we assume that it is related to the grasshoppers' carbohydrate, protein and water requirements. In contrast, vegetative height, which varies independently of tissue quality (Gross et al. 2007), is a reliable indicator of biomass production at the site (Lavorel et al. 2011), and may also influence the grasshoppers' vertical position in the vegetation. We therefore focused on the LES as nutritional value of leaves and VPH as a control trait which should be related only to the habitat of grasshoppers and not to their preferences. Given this general framework, we used the CWM and FD of the LES of the diet, the habitat and the preferences to track the mechanisms that account for the diet of grasshoppers (Fig. 1).

\section{Assessing the diet of grasshoppers from faeces}

We studied five plots within a $13-\mathrm{km}^{2}$ subalpine grassland landscape that ranged in altitude from 1,650 to $1,900 \mathrm{~m}$ a.s.l., within the municipality of Villar d'Arène in the French Alps near the Lautaret Pass. Four grasshopper species (Chorthippus scalaris, Arcyptera fusca, Euthystira brachyptera, Stenobothrus lineatus) representing $43 \pm 18 \%$ of the total number of grasshopper individuals in the five plots studied (Moretti et al. 2013) were sampled in each plot at the end of July and at the beginning of September 2008. For each species, five adult males and five adult females were sampled in each plot and brought back 
to the laboratory in a corked tube. In some cases, more than five individuals of each sex were found, so that a total of 149 individuals were sampled. After a few hours, nearly all of them had produced faeces in the tubes, which were preserved dry, at room temperature, in an Eppendorf tube containing silica gel. Faeces smaller than $2 \mathrm{~mm}$ long originating from the same species, sex and plot were grouped. Thus, we gathered a total of 83 sets of faeces $(37,30,13,2$ and 1 sets containing faeces from 1,2,3, 4 and 5 individuals from the same species, respectively). All DNA extractions were performed in a room dedicated to the extraction of nucleic acids to avoid contamination. The total DNA amount was extracted with the DNeasy Blood and Tissue Kit (QIAgen, Hilden, Germany), following the manufacturer's instructions. The DNA extracts were recovered in a total volume of $250 \mu \mathrm{L}$. Mock extractions without samples were systematically performed to monitor possible contamination. DNA amplifications were carried out on a final volume of $50 \mu \mathrm{L}$, using $4 \mu \mathrm{L}$ of DNA extract as template. The amplification mixture contained $1 \mathrm{U}$ of AmpliTaq Gold DNA Polymerase (Applied Biosystems, Foster City, CA), $10 \mathrm{mM}$ tris(hydroxymethyl)aminomethane- $\mathrm{HCl}, 50 \mathrm{mM} \mathrm{KCl}, 2 \mathrm{mM}$ of $\mathrm{MgCl}_{2}, 0.2 \mathrm{mM}$ of each deoxyribonucleotide triphosphate, $0.1 \mu \mathrm{M}$ of each primer, and $0.005 \mathrm{mg}$ of bovine serum albumin (Roche Diagnostic, Basel, Switzerland). The mixture was denatured at $95{ }^{\circ} \mathrm{C}$ for $10 \mathrm{~min}$, followed by 45 cycles of $30 \mathrm{~s}$ at $95{ }^{\circ} \mathrm{C}$, and $30 \mathrm{~s}$ at $55^{\circ} \mathrm{C}$; as the target sequences are usually shorter than $100 \mathrm{bp}$, the elongation step was removed. Samples were amplified using four primer pairs (Online resource 1). The first pair ( $g$ and $h$ ) corresponds to a universal approach, and targeted the P6 loop region of the trnL (UAA) intron (Taberlet et al. 2007). In order to increase the resolution of the analysis, three other primer pairs were used. They targeted the first internal transcribed spacer (ITS1) of nuclear ribosomal DNA, for Poaceae (ITS1-F and ITS1Poa-R), for Cyperaceae (ITS1-F and ITS1Cyp-R) and for Asteraceae (ITS1-F and ITS1Ast-R) (Baamrane et al. 2012). All primers were modified by the addition of specific tags on the $5^{\prime}$ end to allow the assignment of sequence reads to the relevant sample (Valentini et al. 2009). All the polymerase chain reaction (PCR) products were tagged identically on both ends. These tags were composed of $\mathrm{CC}$ on the $5^{\prime}$ end followed by nine variable nucleotides that were specific to each sample. The nine variable nucleotides were designed using the oligoTag program (http://www.prabi.grenoble. $\mathrm{fr} / \mathrm{trac} / \mathrm{OBITools}$ ) with at least three differences among the tags, without homopolymers longer than two, and avoiding a $\mathrm{C}$ on the $5^{\prime}$ end. All the PCR products from the different samples were first titrated using capillary electrophoresis (QIAxel; QIAgen) and then mixed together, in equimolar concentration, before the sequencing. The sequencing was carried out on the Illumina/Solexa Genome Analyzer IIx
(Illumina, San Diego, CA) by using the Paired-End Cluster Generation Kit V4 and the Sequencing Kit V4 (Illumina) and following the manufacturer's instructions. A total of 108 nucleotides were sequenced on each extremity of the DNA fragments.

Sequence analysis and taxon assignation

The sequence reads were analysed using the OBITools (http://www.prabi.grenoble.fr/trac/OBITools). First, the direct and reverse reads corresponding to a single molecule were aligned and merged using the solexaPairEnd program, taking into account the quality of the data during the alignment and the consensus computation. Then, primers and tags were identified using the ngsfilter program. Only sequences with perfect matches on tags and a maximum of two errors on each primer were considered. The amplified regions, excluding primers and tags, were kept for further analysis. Strictly identical sequences were clustered together using the obiuniq program, keeping the information about their distribution among samples. For the P6 loop of the $t r n \mathrm{~L}$ intron, sequences shorter than $10 \mathrm{bp}$, or with 100 or fewer occurrences, were excluded using the obigrep program. Similarly, for the three ITS1 fragments, sequences shorter than $50 \mathrm{bp}$, or with ten or fewer occurrences, were also excluded. The obiclean program was then implemented to detect amplification/sequencing errors, by giving each sequence within a PCR product the status of "head" (the most common sequence among all sequences that can be linked with a single indel or substitution), "singleton" (no other variant with a single difference in the relevant PCR product), or "internal" (all other sequences that are neither head nor singleton, i.e. sequences owing to amplification/ sequencing errors). Taxon assignation was achieved using the ecoTag program (Pegard et al. 2009). EcoTag relies on a dynamic programming global alignment algorithm (Needleman et al. 1970) to find highly similar sequences in the four reference databases that correspond to the four amplified DNA fragments. These reference databases were built by extracting from the European Molecular Biology Laboratory (EMBL) nucleotide library the relevant part of the chloroplast $t r n \mathrm{~L}$ intron or of the nuclear internal transcribed spacer 1 (ITS1) region using the ecoPCR program (Bellemain et al. 2010). A unique taxon was assigned to each unique sequence. This unique taxon corresponds to the last common ancestor node in the National Center for Biotechnology Information taxonomic tree of all the taxids of the sequences of the reference database that matched the query sequence. Automatically assigned taxonomic identifications were then manually examined to further eliminate a few sequences that had likely resulted either from PCR artefacts or that did not correspond to any plant P6 loop or ITS1 sequences present in the EMBL database (homology 
$<0.9$ ). Sequences with a total of fewer than 100 occurrences of the P6 loop and ITS1 (Poaceae), or fewer than ten for ITS1 (Asteraceae) were also removed. Finally, the comparison between (1) the identification originating from the database built from EMBL, and (2) the list of plant species occurring within the study sites, enabled improved final identification by restricting the final identification to the taxa that are known to be present in the area. A single summary table was compiled based on the data obtained with the four DNA-based identification systems. This table gives the list of identified plant taxa for all 83 analysed samples of faeces and for the four DNA markers separately, ordered according to their apparent relative abundance rank. The relative abundance was estimated according to the number of occurrences of each DNA sequence in the 83 samples. The synthesis of these four parallel scans allowed us to reconstitute the semi-quantitative plant diet for each homogeneous sample corresponding to one grasshopper species in a given plot and at a given date. The DNA-based diet information was then pooled by species (four species), sex (two sexes) and plot (five plots). We transformed the semi-quantitative DNA-based diet data into purely quantitative data according to the procedure described in Online resource 2.

\section{Cafeteria experiment to assess food preferences}

In June 2010, about 20 grasshopper nymphs of each species were collected in the field at the same plots where diet and habitat had been assessed. They were brought into a cage (length $=8 \mathrm{~m}$, width $=3 \mathrm{~m}$, height $=2 \mathrm{~m}$ ), covered by an insect mesh (Boddingtons, Germany), that contained eighteen 40-cm-diameter pots. The pots were filled with intact mesocosms of soil and living plants that were collected in the studied plots. All the 24 species further tested in the cafeteria experiment were present in the cage. Since grasshoppers are capable of learning (Bernays and Bright 2005), we forced them to experience all the plant species occurring in the study area during their last nymphal stages for at least 15 days before the cafeteria experiment, until they became adults. The day before the experiment, fresh and intact leaves of 24 plant species representative of the communities in the study area (Online resource 3) were collected in the field and hydrated in a beaker during one night in dark and cool conditions. The selected species were either dominant ( $>20 \%$ of biomass) or subdominant ( $>10 \%$ of biomass) in the study area, and their functional traits covered the functional range of the natural communities (Lavorel et al. 2008). Leaves were randomly placed into $40 \times 20 \times 4$-cm boxes closed with a cover and further hydrated to prevent withering. The water content is the only functional trait that might be different from field conditions (Garnier et al. 2001), although leaves were hydrated following harvest and throughout the experiment. Single adult grasshoppers (five females and five males of four studied species) were collected from the greenhouse, starved for one night at $5{ }^{\circ} \mathrm{C}$, which corresponds to the overnight field temperature, and then sequentially introduced into the box. After $5 \mathrm{~h}$, each leaf was checked for signs of herbivory, and if there were any, the percentage eaten was estimated visually, always by the same person (Q. Duparc). The area eaten was then estimated by multiplying the percentage of the leaf eaten by the mean area of nine leaves of the plant species measured before the experiment. The proportion of the leaf area of each plant species eaten by the five grasshoppers of each species and sex was pooled and standardized to sum to one. The final data set used in the following analysis comprises eight vectors of plant preferences, one for each species and sex.

\section{Plant traits and calculation of diet values}

Floristic composition of dominant and subdominant species in each grassland plot was assessed using the Botanal method, providing species relative abundances (Lavorel et al. 2008). The six plant traits studied (SLA, LDMC, LNC, LCC, LPC, and VPH) were measured for the species making up $80 \%$ of the total cumulated abundance using standardised protocols (Cornelissen et al. 2003). The complete set of six traits was used in multivariate analyses. In univariate analyses we used only LES and VPH in order to avoid redundant information, as these two traits are almost orthogonal being collinear to the first two axes of a PCA including all five traits (not shown). For both LES and VPH, we calculated the CWM and the FD of each of the five field plots. CWM was calculated following (Lavorel et al. 2008):

$\mathrm{CWM}=\sum_{i=1}^{n} p_{i} \times$ trait $_{i}$

where $n$ is the total number of plant species, $p_{i}$ the relative abundance of species $i$ and trait ${ }_{i}$ the trait value of species $i$. FD was calculated as the mean distance of the species' traits to the CWM (Laliberté and Legendre 2010). Likewise, we then calculated the CWM and FD of preferences for both sexes of each of the four grasshopper species, using the relative proportion of plant species consumption in the cafeteria experiment. Finally, we calculated the CWM and FD of the diets for both sexes of each of the four species in each of the five plots, using the estimated relative abundance of plant species in the faeces (Online resource 5).

Data analysis

All data handling and statistical analyses were carried out with R Development Core Team software (2011). The data 
set available for the analyses contained unique combinations of sex, grasshopper species and plots, which were described by (1) traits' CWM and FD for their realised diet values, (2) traits' CWM and FD of preference for each sex per species combination, and (3) traits' CWM and FD for habitat values in each plot.

\section{Diet differentiation across species and plots (prediction 1)}

For LES and VPH, we used a Tukey's honestly significant difference (HSD) test for all pairs of species, as well as for all pairs of plots to test for diet differentiation across species and plots. We then performed linear discriminant analysis on the six traits to obtain a graphical representation of the diet differentiation between grasshopper species or plots, respectively. Linear discriminant analysis is similar to PCA, but instead of maximizing the overall variance, axes maximize the variance between groups, in our case species or plots, respectively.

\section{Variation of the diet explained by habitat and preferences (prediction 2)}

To assess the relative effect of habitat and preferences on the diet of the four grasshopper species, we used linear mixed models of the diet value with the habitat and the preference values of LES or VPH as fixed effects. The random effects were sex nested into species and plots. Four models were calculated: (1) the full model comprising both the habitat and preferences effect which tested for joint effects of habitat and preferences on diet, (2) the habitat model which tested whether diet depended only on habitat availability of plant material regardless of grasshoppers' preferences, (3) the preferences model which tested the whether diet depended only on preferences expressed in the cafeteria experiment regardless of plant availability in the field, and (4) the null model including the random effects only, i.e. assuming that neither availability nor preferences were relevant to consumption in the field. The models were calculated using the maximum likelihood criterion in order to compare their corrected Akaike information criteria (AICc). For each model, we calculated the difference $(\triangle \mathrm{AICc})$ between its own AICc and the model having the lowest AICc. We then selected the models with $\triangle \mathrm{AICc}<2$. Among these models, we finally chose the most parsimonious one to conclude whether variation in the habitat and/or the preferences affected diet.

\section{Results}

Plant species composition of diet and preferences

In the following analyses, we retained 34 plant species that were observed at least three times in the faeces out of 74 species found at least once (Online resource 4), while excluding Triticum sp. and Larix decidua since they were not present in the plots. These 34 plant species represented $92 \%$ of the sequence reads after filtering. Among the 326 different DNA fragments detected and that corresponded to these 34 plant species, $263(81 \%)$ belonged to graminoid species, 20 (6\%) to legumes and $43(13 \%)$ to other dicots. The four grasshopper species did not have the same feeding pattern with respect to plant families (Table 1). For example, Stenobothrus lineatus almost only ate grasses (Poaceae and Cyperaceae) whereas about one-third of the fragments found in the faeces of Chorthippus scalaris belonged to dicots. C. scalaris was the most generalised species (23 plant species eaten both by males and females) while $S$. lineatus had a less diversified diet (seven species eaten by males, 13 by females; Table 1 ). The complete data corresponding to the relative abundance of each of the 34

Table 1 Estimated percentage eaten of the different plant families by each grasshopper species and sex across all plots

\begin{tabular}{|c|c|c|c|c|c|c|c|}
\hline \multirow[t]{2}{*}{ Grasshoppers } & \multirow[t]{2}{*}{ Sex } & \multicolumn{5}{|c|}{ Percentage eaten } & \multirow[t]{2}{*}{ Degree $^{b}$} \\
\hline & & Poaceae & Cyperaceae & Fabaceae & Asteraceae & Other dicots ${ }^{\mathrm{a}}$ & \\
\hline \multirow[t]{2}{*}{ Arcyptera fusca } & Female & 85.7 & 2.0 & 0.0 & 9.6 & 2.8 & 18 \\
\hline & Male & 79.3 & 0.8 & 4.7 & 11.7 & 3.5 & 17 \\
\hline \multirow[t]{2}{*}{ Chorthippus scalaris } & Female & 62.2 & 5.2 & 18.4 & 11.1 & 3.1 & 23 \\
\hline & Male & 60.0 & 6.8 & 13.0 & 10.7 & 9.6 & 23 \\
\hline \multirow[t]{2}{*}{ Euthystira brachyptera } & Female & 85.7 & 4.8 & 1.8 & 3.1 & 4.6 & 22 \\
\hline & Male & 92.7 & 2.8 & 0.0 & 1.1 & 3.4 & 16 \\
\hline \multirow[t]{2}{*}{ Stenobothrus lineatus } & Female & 87.7 & 11.5 & 0.0 & 0.0 & 0.8 & 13 \\
\hline & Male & 91.3 & 8.7 & 0.0 & 0.0 & 0.0 & 7 \\
\hline
\end{tabular}

See Electronic supplementary material 2 for the estimation of the percentage eaten from the DNA fragments data

a Include Apiaceae, Campanulaceae, Cistaceae, Liliaceae and Orobanchaceae

b Corresponds to the number of plant species eaten 
plant species in the faeces of each available combination of sex, grasshopper species and plot are presented in Online resource 5. Among the 24 plant species tested in the cafeteria experiment, 19 were present in the faeces, representing $232(71 \%)$ DNA fragments out of the 326 fragments considered in the analysis. In the cafeteria experiment, 16 (67\%) species out of the 24 species used were eaten by the four studied grasshopper species. Graminoid species were preferred (58 \% of the eaten leaf area), followed by legumes $(27 \%)$ and other forbs $(14 \%)$. There was no correlation between the area eaten and the mean area of individual leaves (Pearson's product-moment correlation $r=-0.04$, $d f=22, P=0.81$ ), excluding the confounding effect of the leaf area in the cafeteria experiment.

LES values of the plant species and in the diet

The LES corresponds to the first axis of a PCA including SLA, LNC and LDMC, which explained $99 \%$ of the variance of these three traits. The scores of the three traits on the first axis (i.e. LES) were 0.78 (SLA), 0.82 (LNC) and -0.86 (LDMC). Therefore, the leaves of plant species having high LES values were thin, and rich in nitrogen and water, whereas plant species with low LES values were dense, and poor in nitrogen and water. The Festuca genus had the lowest LES values (about -2), as well as most

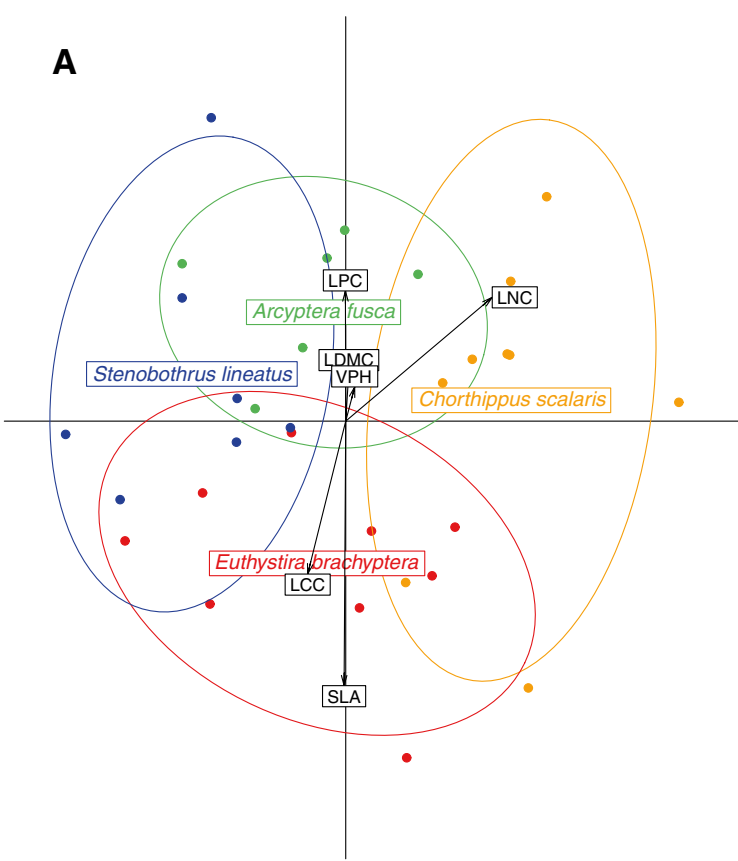

Fig. 2 Diet differentiation based on the mean (CWM) of the six leaf traits [specific leaf area $(S L A)$, leaf dry matter content $(L D M C)$, leaf nitrogen content $(L N C)$, leaf carbon content $(L C C)$, leaf phosphorus content $(L P C)$, and vegetative plant height $(V P H)]$ across a the four grasshopper species, and $\mathbf{b}$ the five plots evidenced by a linear discri- grasses, but some grasses showed intermediate (e.g. -0.08 , Dactylis glomerata and -0.2 , Trisetum flavescens) to high (1.35, Anthoxanthum odoratum) LES values (Electronic supplementary material 4; ESM 4). Vicia cracca (Fabaceae) had the highest LES values (4.22), and most dicots and high LES values, except Achillea millefolium (-1.09) and Helianthemum grandifolium (-0.40). The LES values of the diets covered the whole range of the available traits in the habitats. Festuca species having a LES value lower than -2 occurred 51 times in the faeces (17\% of the occurrences), while species having a LES values lower than -1 occurred 172 times (53\% of the occurrences). Plant species having a LES value $>1$ occurred 35 times ( $11 \%$ of the occurrences). $V$. cracca, the species with the highest LES value, occurred five times in the faeces ( $2 \%$ of the occurrences; ESM 4 ).

Diet differentiation across grasshopper species and plots (prediction 1)

Four out of the six possible grasshopper species pairs showed significant differences in their diet for LES (Tukey HSD tests; see also Fig. 3a); the two pairs showing no differences were E. brachypteralA. fusca, and $S$. lineatus/A. fusca. None showed significant differences in their diet for VPH. The diet of $C$. scalaris was characterised by leaves rich in nitrogen (Fig. 2a) with a relatively

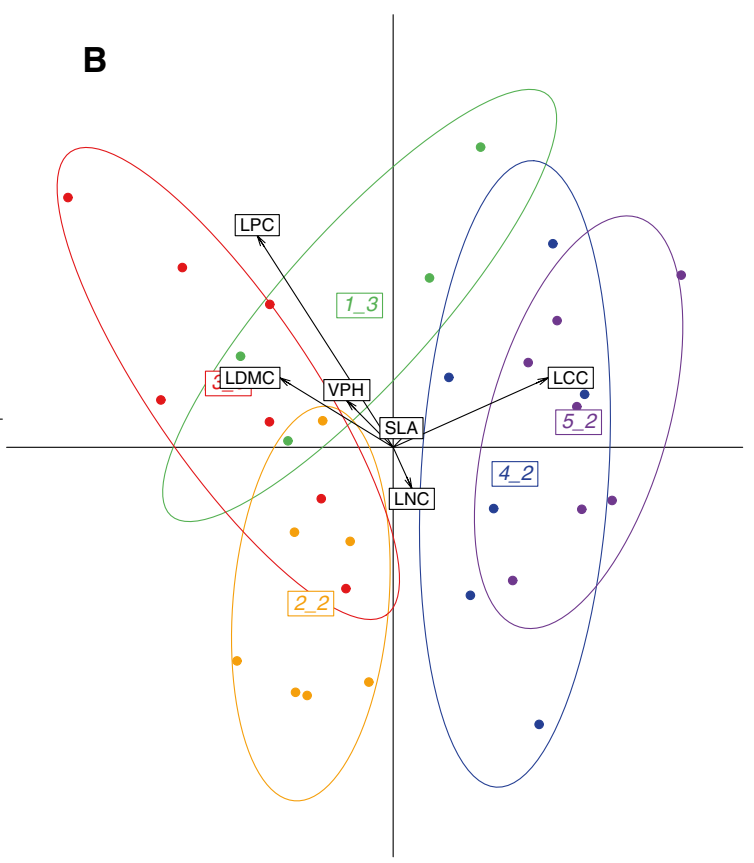

minant analysis, which maximizes differences between groups. Each point corresponds to a sex by species in one of the five plots studied. The $x$-axis corresponds to the first axis of the discriminant analysis, the $y$-axis corresponds to the second axis 

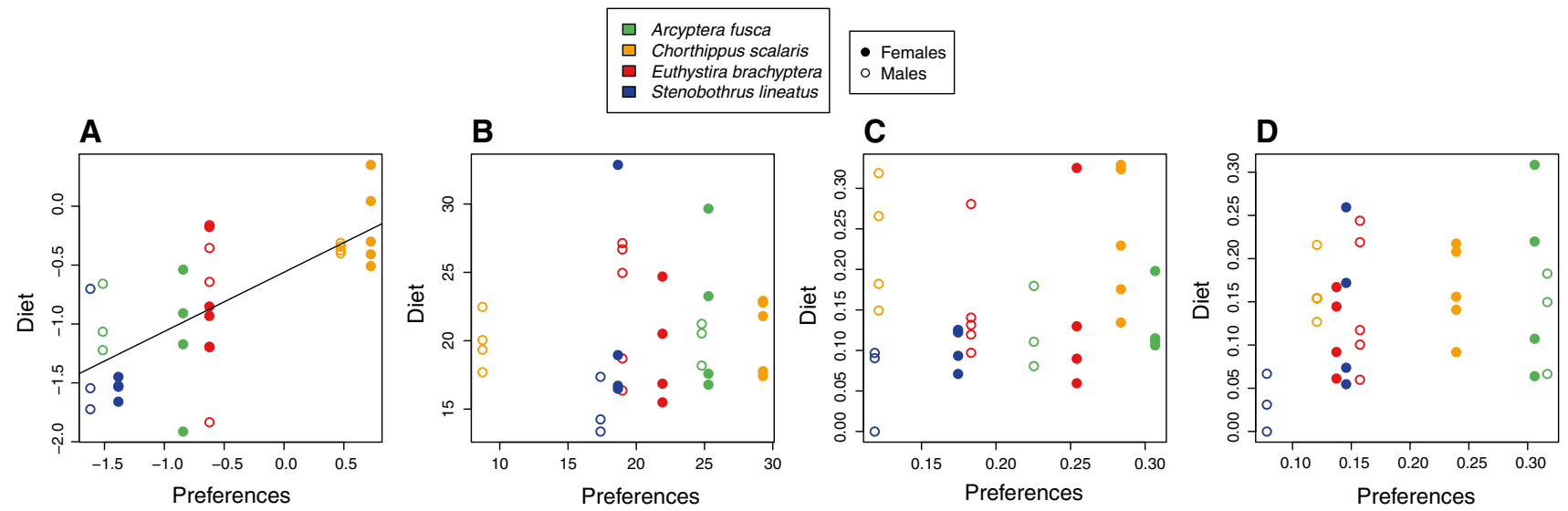

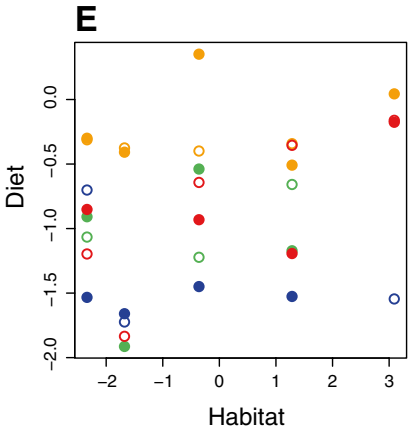

Leaf economics spectrum

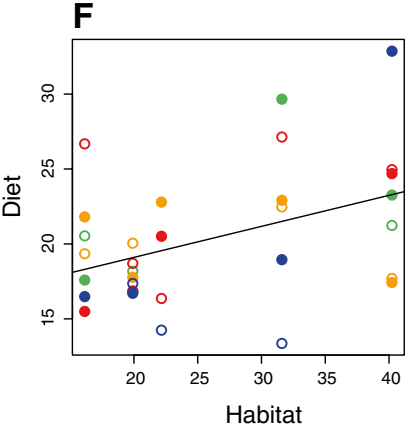

Vegetative height

Community weighed means

Fig. 3 Relative contribution of preferences and habitat to the diet. a, b CWM of the leaf economic spectrum (LES) (see "Materials and methods" for details) and the VPH in the diet as a function of the corresponding CWM in the preferences. c, $\mathbf{d}$ FD of LES and VPH in the diet as a function of the corresponding FD in the preferences. $\mathbf{e}-\mathbf{h}$

high LES value (Fig. 3a), S. lineatus ate leaves with a poorer nitrogen content (Fig. 2a) and a low LES value (Fig. 3a). The two other species, E. brachyptera and A. fusca, showed intermediate values. In contrast, none of the ten plot pairs showed significant differences, neither for LES nor for VPH (Fig. 2b).

Variation in the diet explained by habitat and preferences (prediction 2)

When considering mean trait values (CWM) for the variation in the grasshoppers' diet, for the LES the most parsimonious model showed that only differences in preferences were relevant for the variation (Table 2; Fig. 3). In contrast, for VPH variation in the diet was most parsimoniously explained by differences in habitats (Table 2; Fig. 3). When considering FD, the most parsimonious model showed that only differences in the habitat LES accounted for variation in the diet LES (Table 2; Fig. 3). For VPH, the model that included both preferences and habitat had the lowest

\section{G}

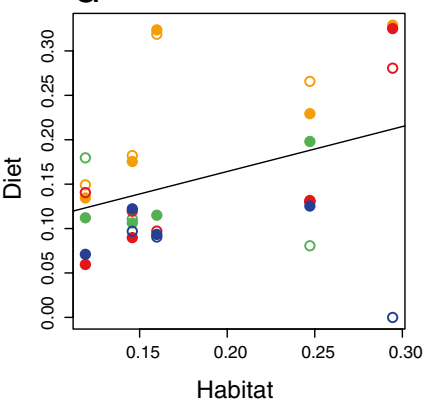

Leaf economics spectrum

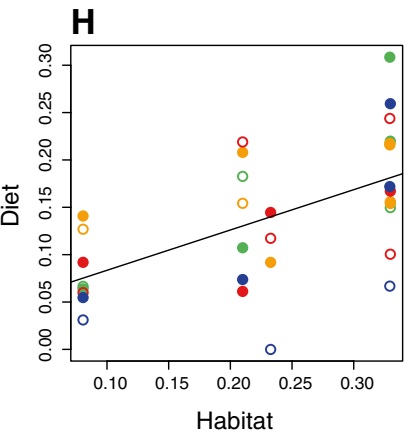

Vegetative height

Functional diversity

Plots are similar to plots $\mathbf{a}-\mathbf{d}$, except that the habitat values are given on the $x$-axis. Each point corresponds to a sex by species in a single plot. The presence of a correlation line indicates that the relationship between the two variables is significant, on the basis of linear mixed models. For other abbreviations, see Figs. 1 and 2

AICc value, but it was closely followed by the model that included only habitat (Table $2 ; \Delta \mathrm{AICc}=0.66$ ).

\section{Discussion}

A new technique to study the diet of grasshoppers

To our knowledge, this is the first community-level study of grasshopper diet using faeces as a source of DNA, and one of the first with insect herbivores (Jurado-Rivera et al. 2009; Staudacher et al. 2011). Until recently, the only available techniques for the study of a herbivore's diet were the identification under the microscope of plant fragments found in the gut after dissection or in the faeces (Joern 1979), the direct observation of the behaviour of herbivores (Singer and Stireman 2001) and the use of carbon isotope ratios (Fry et al. 1978). Depending on the traits of the plants and grasshoppers in question, and on the grasshopper physiological status, leaves are subject 
Table 2 Niche position (community weighted mean; CWM) and niche breadth (functional diversity; FD) of the vegetative plant height $(V P H)$ and the leaf economic spectrum $(L E C)$ predicted by linear mixed models

\begin{tabular}{lll}
\hline & VPH & LES \\
\hline CWMs & & \\
Full model & 2.95 & 0.86 \\
Preferences & 5.26 & $0^{\mathrm{a}}$ \\
Habitat & $0^{\mathrm{a}}$ & 6.85 \\
Null model & 2.52 & 6.11 \\
FD & & \\
Full model & 0 & 3.1 \\
Preferences & 4.71 & 5.19 \\
Habitat & $0.66^{\mathrm{a}}$ & $0^{\mathrm{a}}$ \\
Null model & 4.84 & 2.41 \\
\hline
\end{tabular}

For each plant trait, four models are computed: the full model comprising both the habitat and the preference effects, the habitat model, the preference model, and the null model including the random effects only (species-sex nested into species; and plot). The presented data are corrected Akaike information criteria $(\triangle \mathrm{AICc})$; they correspond to the AICc of each of the four models minus the lowest AICc, so that the value 0 indicates the model with the lowest AICc

${ }^{a}$ The selected model, which corresponds to the most parsimonious model among the models having a $\triangle \mathrm{AICc}<2$ (in italics)

to differential fragmentation by insect mandibles and to differential digestion in the gut, which leads to potential biases when the diet is estimated under the microscope. Microhistological analyses using faeces is biased towards taxa with a resistant cuticle (Leslie et al. 1983), such as ferns, sedges and grasses. The DNA barcoding technique also has its limitations, both for technical and biological reasons (Pompanon et al. 2012). Due to the DNA amplification process, the technique is only semi-quantitative, for example it cannot completely discriminate plants briefly sampled by grasshoppers vs. plants that constitute a minor but significant part of their diet. In addition, the DNA extractability depends on the morphology and the toughness of cell walls, which depend on species, tissues and phenology. In future studies, cafeteria assays could be used in combination with DNA barcoding of the faeces to explore these limitations in more details. However, the DNA barcoding method appears to be more reliable, as suggested by a recent study comparing microhistology with DNA analysis of rodent stomach contents (Soininen et al. 2009) where the DNA approach provided a much better taxonomic resolution, while preserving the relative proportions among plant taxa. Furthermore, the DNA technique has the advantage of being less time-consuming than the microscope technique-though it is more costly. The use of DNA barcoding is therefore promising for the study of large herbivory networks. As expected, the diets of the four Gomphocerinae grasshopper species were both dominated by grasses and mixed with legume species and other forbs, which is consistent with previous studies (Joern 1979; Franzke et al. 2010). From a family-level perspective, the studied grasshoppers could therefore be seen as grass specialists. However, the different grass species eaten were functionally contrasted (ESM 3 and 4), ranging from species with a very low LES (e.g. Festuca spp.) to species having a LES similar to or even higher than many dicots (e.g. Anthoxanthum odoratum), so we instead adopted a functional approach, according to which the four studied species have a generalized diet.

\section{Preferences drive niche position}

Our results clearly showed that the mean value (CWM) for plant nutritional value (i.e. LES) in the diet was explained by food preferences, but not by habitat (Fig. 3). In a study focusing on the identity of the plant species eaten, Bernays and Chapman (1970) found that the diet of Chorthippus paralellus was correlated to grass abundances in the field. The plant selection process by grasshoppers would then involve two steps: (1) focus on the plant species having the preferred functional traits and (2) among these species, choose the most abundant. When considering mean value for plant stature (VPH) in the diet this was, as expected, correlated to the habitat and not to the food preferences. There are two possible reasons for this: (1) VPH is a proxy for plant biomass (Lavorel et al. 2011), which reflects the fact that abundant plants are consumed in greater amounts, and (2) the cafeteria experiment was designed to avoid effects from plant architecture, which excludes VPH as a selection criteria from the preferences. Hence, from a methodological view point, cafeteria experiments provide valuable information on the diet of herbivores when relevant plant traits, such as LES or other related nutritional traits, are considered (PérezHarguindeguy et al. 2003).

Since the habitat had no influence on the mean characteristics (CWM) of the plant nutritional quality (LES) in the diet, why then did the diet not match food preferences perfectly? There are three possible reasons for this: (1) other functional traits beside plant nutritional values might govern plant-herbivore interactions, such as biomechanical traits (e.g. Peeters et al. 2007), and secondary compounds in plants (Bernays and Chapman 1994), (2) predators modify the location of herbivores in the herbaceous strata, which leads to a sub-optimal diet (e.g. Orrock et al. 2004), (3) herbivores have well-defined micro-habitat preferences (Joern 1982), so that their food and shelter niches can be independent of each other (Orav-Kotta and Kotta 2004), leading to an absence of a relationship between mean characteristics of habitat and diet, as we found here. 
Habitat drives diet mixing

Our results showed that for both LES and VPH, the habitat rather than the preferences explained the variation of FD in the diet (Fig. 3). This pattern contrasts with that for mean (CWM) LES in the diet, which was explained by the preferences alone. In functionally diverse plant communities, such as the ones studied here, the plant FD in the diet of the different grasshopper species is so large that their niches overlap. The influence of the habitat FD on the diet FD is therefore likely to be a mechanism that limits niche partitioning. This FD of the diet can be interpreted as diet mixing, i.e. when grasshoppers consume plants with complementary traits. In our case, the diet FD for the plants' nutritional value can be assumed to correspond to optimal nutrition, but herbivores can have mixed diets for other purposes, e.g. to avoid ingesting large amounts of secondary compounds or to become "vaccinated" against predators (Bernays et al. 1994; Unsicker et al. 2008). Here, the diet-mixing process was determined primarily by the availability of different plants in the habitat, where more diverse habitats, rather than preferences, led to more diverse diets (Joern 1979). This means that the level of functional generalization of herbivores was not an intrinsic requirement of species but rather of habitat context (Behmer et al. 2002; Raubenheimer and Simpson 2003; Simpson et al. 2004). This can be of particular interest for the study of plant-herbivore interaction webs, where the generalization level is usually implicitly considered as species-specific (Thébault and Fontaine 2008). Nevertheless, the mechanisms through which habitat influences diet mixing remain unclear. Do grasshoppers actively look for plants with diverse functional characteristics, or does a mixed diet simply result from random encounters with diverse suitable plants? Dietary mixing may lead to a higher fitness for grasshoppers living in more diverse habitats (Bernays et al. 1994; Unsicker et al. 2010), but in the habitats we studied here, all the main functional groups of plants (conservative and exploitative grasses, legumes, other forbs; Quétier et al. 2007) were present, which is likely to be sufficient for the grasshoppers to achieve high fitness even by chance encounters (Specht et al. 2008).

\section{Niche partitioning}

It has been suggested that interspecific competition between herbivores that share common resources leads to niche partitioning (e.g. Belovsky 1986). This appears to be also true for generalist grazers that consume the same plant species but in different proportions (Behmer and Joern 2008), like in our study. We found that, when mean values (CWM) of the LES were considered, the diets of four out of six grasshopper species pairs were significantly different, as shown by Tukey HSD tests and illustrated in Fig. 2, where the niches of the four grasshopper species are partitioned, albeit with large overlaps. Cafeteria experiments with artificial food by Behmer and Joern (2008) showed that different species of generalist grasshoppers had contrasting preferences for food items containing various carbohydrate/ protein ratios, i.e. contrasting nutritional preferences. Our study goes one step further and strongly suggests that (1) the differences in the nutritional preferences among species observed by Behmer and Joern (2008) are reflected in differences in food selection based on plant functional traits, and (2) the interspecific differences in food preferences are reflected in the contrasts between diets of co-occurring grasshopper species in the field. Our results, combined with previous findings, thus suggest a mechanistic explanation for dietary differentiation among species based on three hierarchical levels: (1) nutritional preferences for a given protein:carbohydrate ratio as expressed with artificial food (Simpson and Raubenheimer 1993; Behmer and Joern 2008), (2) preferences for plant species as expressed in choice experiments, and (3) actual diet ultimately realised in situ. However, we found a large overlap between species' diets (Fig. 2) whereas Behmer and Joern (2008) found nearly no overlap between species' nutritional preferences (their Fig. 1). Hence, the existence of unique nutritional preferences does not necessarily lead to strict niche partitioning in nature, presumably because the nutritional properties of the available plants are less contrasted than experimental food items, and because a complex combination of factors determines plant selection in nature, as outlined in the previous section. In contrast to the diet differences across species, we found no significant differences across plots (Fig. 2), although these clearly differed in functional composition (Quétier et al. 2007), highlighting that the niche position based on the LES of the dominant plant species (CWM) in the diet is principally determined by preferences.

\section{Conclusion}

Our study is the first to assess the diet of grasshoppers using faeces as a source of DNA, and to compare it simultaneously with the grasshoppers' feeding preferences and with the availability of plants in their habitat. Two major conclusions shed light on the mechanisms that account for the diet of grasshoppers. First, the nutritional niche position (i.e. the CWM of LES) depends on species-specific preferences, which is the basis for niche partitioning between species, a central issue for diet comparisons across herbivore species. Second, niche breadth (i.e. the FD of LES) depends on the diversity of available plants. Based on these results, the level of generalisation 
is not an intrinsic property of a given species, as generally considered in interaction network studies, but rather results from complex interactions between the preferences of a species and its habitat. We conclude that future studies should consider jointly niche position and niche breadth for the study of the factors determining interaction niches.

Acknowledgments We are grateful to Simone Fontana, Stéphane Bec and Louise Boulangeat for their help during field work, to Rolland Douzet for his help with plant identification, and to Kurtis Gautschi for his help with the English language. Comments from two anonymous reviewers allowed significant improvements of the manuscript. This research was conducted on the long-term research site Zone Atelier Alpes (ZAA), part of the International Long-Term Ecological Research-Europe network. This work is ZAA publication no. 26.

Data accessibility The Fasta file and filtered data of the DNAbased diet analysis are deposited in the Dryad repository: http:// doi.org/10.5061/dryad.fr0pd.

\section{References}

Asshoff R, Hattenschwiler S (2005) Growth and reproduction of the alpine grasshopper Miramella alpina feeding on $\mathrm{CO}_{2}$-enriched dwarf shrubs at treeline. Oecologia 142:191-201

Baamrane MAA, Shehzad W, Ouhammou A, Abbad A, Naimi M, Coissac E, Taberlet P, Znari M (2012) Assessment of the food habits of the Moroccan dorcas gazelle in M'Sabih Talaa, West central Morocco, using the trnL approach. PLoS One 7:e35643

Behmer ST (2009) Insect herbivore nutrient regulation. Annu Rev Entomol 54:165-187

Behmer ST, Joern A (2008) Coexisting generalist herbivores occupy unique nutritional feeding niches. Proc Natl Acad Sci 105:1977-1982

Behmer ST, Simpson SJ, Raubenheimer D (2002) Herbivore foraging in chemically heterogeneous environments: nutrients and secondary metabolites. Ecology 83:2489-2501

Bellemain E, Carlsen T, Brochmann C, Coissac E, Taberlet P, Kauserud H (2010) ITS as an environmental DNA barcode for fungi: an in silico approach reveals potential PCR biases. BMC Microbiol 10:189

Belovsky GE (1986) Generalist herbivore foraging and its role in competitive interactions. Am Zool 26:51-69

Bernays EA, Bright KL (2005) Distinctive flavours improve foraging efficiency in the polyphagous grasshopper, Taeniopoda eques. Anim Behav 69:463-469

Bernays EA, Chapman RF (1970) Food selection by Chorthippus parallelus (Zetterstedt) (Orthoptera: Acrididae) in the field. J Anim Ecol 39:383-394

Bernays EA, Chapman RF (1994) Host plant selection by phytophagous insects. Springer, New York

Bernays EA, Bright KL, Gonzalez N, Angel J (1994) Dietary mixing in a generalist herbivore: tests of two hypotheses. Ecology 75:1997-2006

Cornelissen JHC, Lavorel S, Garnier E, Díaz S, Buchmann N, Gurvich $\mathrm{DE}$, Reich $\mathrm{PB}$, ter Steege $\mathrm{H}$, Morgan $\mathrm{HD}$, van der Heijden MGA, Pausas JG, Poorter H (2003) A handbook of protocols for standardised and easy measurement of plant functional traits worldwide. Aust J Bot 51:335-380

Franzke A, Unsicker SB, Specht J, Koehler G, Weisser WW (2010) Being a generalist herbivore in a diverse world: how do diets from different grasslands influence food plant selection and fitness of the grasshopper Chorthippus parallelus? Ecol Entomol 35:126-138

Fry B, Joern A, Parker PL (1978) Grasshopper food web analysis: use of carbon isotope rates to examine feeding relationships among terrestrial herbivores. Ecology 59:498-506

Garnier E, Shipley B, Roumet C, Laurent G (2001) A standardized protocol for the determination of specific leaf area and leaf dry matter content. Funct Ecol 15:688-695

Garnier E, Cortez J, Billès G, Navas M-L, Roumet C, Debussche M, Laurent G, Blanchard A, Aubry D, Bellmann A, Neill C, Toussaint J-P (2004) Plant functional markers capture ecosystem properties during secondary succession. Ecology 85:2630-2637

Gross N, Suding KN, Lavorel S (2007) Leaf dry matter content and lateral spread predict response to land-use change for six subalpine grassland species. J Veg Sci 18:289-300

Joern A (1979) Feeding patterns in grasshoppers (Orthoptera: Acrididae): factors influencing diet specialization. Oecologia 38:325-347

Joern A (1982) Vegetation structure and microhabitat selection in grasshoppers (Orthoptera: Acrididae). Southwest Nat 27:197-209

Jurado-Rivera JA, Vogler AP, Reid CAM, Petitpierre E, Gómez-Zurita J (2009) DNA barcoding insect-host plant associations. Proc R Soc B Biol Sci 276:639-648

Laliberté E, Legendre P (2010) A distance-based framework for measuring functional diversity from multiple traits. Ecology 91:299-305

Lavorel S, Grigulis K, McIntyre S, Williams NSG, Garden D, Dorrough J, Berman S, Quetier F, Thebault A, Bonis A (2008) Assessing functional diversity in the field-methodology matters! Funct Ecol 22:134-147

Lavorel S, Grigulis K, Lamarque P, Colace M-P, Garden D, Girel J, Pellet G, Douzet R (2011) Using plant functional traits to understand the landscape distribution of multiple ecosystem services. $\mathbf{J}$ Ecol 99:135-147

Leslie DM Jr, Vavra M, Starkey EE, Slater RC (1983) Correcting for differential digestibility in microhistological analyses involving common coastal forages of the Pacific Northwest. J Range Manage 36:730-732

Moretti M, De Bello F, Ibanez S, Fontana S, Pezzatti B, Dziock F, Rixen C, Lavorel S (2013) Linking traits between plants and invertebrate herbivores to track functional effects of environmental changes. J Veg Sci (in press)

Needleman SB, Wunsch CD et al (1970) A general method applicable to the search for similarities in the amino acid sequence of two proteins. J Mol Biol 48:443-453

Ogden J (1976) Some aspects of herbivore-plant relationships on Caribbean reefs and seagrass beds. Aquat Bot 2:103-116

Orav-Kotta H, Kotta J (2004) Food and habitat choice of the isopod Idotea baltica in the northeastern Baltic Sea. Hydrobiologia 514:1-3

Orrock JL, Danielson BJ, Brinkerhoff RJ (2004) Rodent foraging is affected by indirect, but not by direct, cues of predation risk. Behav Ecol 15:433-437

Peeters PJ, Sanson GD, Read J (2007) Leaf biomechanical properties and the densities of herbivorous insect guilds. Funct Ecol 21:246-255

Pegard A, Miquel C, Valentini A, Coissac E, Bouvier F, François D, Taberlet P, Engel E, Pompanon F (2009) Universal DNA-based methods for assessing the diet of grazing livestock and wildlife from feces. J Agric Food Chem 57:5700-5706

Pérez-Harguindeguy N, Díaz S, Vendramini F, Cornelissen JHC, Gurvich DE, Cabido M (2003) Leaf traits and herbivore selection in the field and in cafeteria experiments. Aust Ecol 28: $642-650$ 
Pompanon F, Deagle BE, Symondson WOC, Brown D, Jarman SN, Taberlet $\mathrm{P}$ (2012) Who is eating what: diet assessment using next generation sequencing. Mol Ecol 21:1931-1950

Pontes L, Soussana JF, Louault F, Andueza D, Carrere P (2007) Leaf traits affect the above-ground productivity and quality of pasture grasses. Funct Ecol 21:844-853

Quétier F, Thébault A, Lavorel S (2007) Plant traits in a state and transition framework as markers of ecosystem response to land-use change. Ecol Monogr 77:33-52

R Development Core Team (2011) R: a language and environment for statistical computing. R Foundation for Statistical Computing, Vienna

Raubenheimer D, Simpson SJ (2003) Nutrient balancing in grasshoppers: behavioural and physiological correlates of dietary breadth. J Exp Biol 206:1669-1681

Rowell-Rahier M (1984) The presence or absence of phenolglycosides in Salix (Salicaceae) leaves and the level of dietary specialisation of some of their herbivorous insects. Oecologia 62:26-30

Schädler M, Jung G, Auge H, Brandl R (2003) Palatability, decomposition and insect herbivory: patterns in a successional old-field plant community. Oikos 103:121-132

Simpson SJ, Raubenheimer D (1993) A multi-level analysis of feeding behaviour: the geometry of nutritional decisions. Philos Trans R Soc Lond B 342:381-402. doi:10.1098/rstb.1993.0166

Simpson SJ, Sibly RM, Lee KP, Behmer ST, Raubenheimer D (2004) Optimal foraging when regulating intake of multiple nutrients. Anim Behav 68:1299-1311

Singer M, Stireman J (2001) How foraging tactics determine hostplant use by a polyphagous caterpillar. Oecologia 129:98-105

Soininen EM, Valentini A, Coissac E, Miquel C, Gielly L, Brochmann C, Brysting AK, Sønstebø JH, Ims RA, Yoccoz NG et al (2009) Analysing diet of small herbivores: the efficiency of DNA barcoding coupled with high-throughput pyrosequencing for deciphering the composition of complex plant mixtures. Front Zool 6:16

Specht J, Scherber C, Unsicker SB, Kohler G, Weisser WW (2008) Diversity and beyond: plant functional identity determines herbivore performance. J Anim Ecol 77:1047-1055
Speiser B, Rowell-Rahier M (1991) Effects of food availability, nutritional value, and alkaloids on food choice in the generalist herbivore Arianta arbustorum (Gastropoda: Helicidae). Oikos 62:306-318

Staudacher K, Wallinger C, Schallhart N, Traugott M (2011) Detecting ingested plant DNA in soil-living insect larvae. Soil Biol Biochem 43:346-350

Sword GA, Joern A, Senior LB (2005) Host plant-associated genetic differentiation in the snakeweed grasshopper, Hesperotettix viridis (Orthoptera: Acrididae). Mol Ecol 14:2197-2205

Taberlet P, Coissac E, Pompanon F, Gielly L, Miquel C, Valentini A, Vermat T, Corthier G, Brochmann C, Willerslev E (2007) Power and limitations of the chloroplast $\operatorname{trn} \mathrm{L}$ (UAA) intron for plant DNA barcoding. Nucl Acids Res 35:e14

Thébault E, Fontaine C (2008) Does asymmetric specialization differ between mutualistic and trophic networks? Oikos 117:555-563

Unsicker SB, Oswald A, Kohler G, Weisser WW (2008) Complementarity effects through dietary mixing enhance the performance of a generalist insect herbivore. Oecologia 156:313-324

Unsicker SB, Franzke A, Specht J, Köhler G, Linz J, Renker C, Stein C, Weisser WW (2010) Plant species richness in montane grasslands affects the fitness of a generalist grasshopper species. Ecology 91:1083-1091

Valentini A, Miquel C, Nawaz MA, Bellemain E, Coissac E, Pompanon FCO, Gielly L, Cruaud C, Nascetti G, Wincker P et al (2009) New perspectives in diet analysis based on DNA barcoding and parallel pyrosequencing: the $\operatorname{trn} \mathrm{L}$ approach. Mol Ecol Resour 9:51-60

Wright IJ, Reich PB, Westoby M, Ackerly DD, Baruch Z, Bongers F, Cavender-Bares J, Chapin T, Cornelissen JHC, Diemer M, Flexas J, Garnier E, Groom PK, Gulias J, Hikosaka K, Lamont BB, Lee T, Lee W, Lusk C, Midgley JJ, Navas M-L, Niinemets Ü, Oleksyn J, Osada N, Poorter H, Poot P, Prior L, Pyankov VI, Roumet C, Thomas SC, Tjoelker MG, Veneklaas EJ, Villar R (2004) The worldwide leaf economics spectrum. Nature 428:821-827 